\title{
INTEROBSERVER REPRODUCIBILITY OF QUALITATIVE AND QUANTITATIVE ANALYSIS OF INTRAVASCULAR ULTRASOUND IMAGES BEFORE AND AFTER PERIPIERAL BALLOON ANGIOPLASTY
}

\author{
Aad van der Lugt, Elma J. Gussenhoven, Gerard Pasterkamp, \\ Nicolaas Bom, David J. Posthuma and Theo Stijnen \\ University Hospital Rotterdam-Dijkzigt, Erasmus University Rotterdam, University Hospital Utrecht, \\ The Netherlands Heart Foundation and the Interuniversity Cardiology Institute, The Netherlands
}

\author{
(Received 6 July 1995; in final form 28 December 1995)
}

\begin{abstract}
In this study, interobserver agreement on intravascular ultrasound data obtained before and after balloon angioplasty (PTA) of the superficial femoral artery was assessed. Two observers analyzed intravascular ultrasound cross-sections from 38 patients. Interobserver agreement was good for soft and hard lesions, dissection and vascular damage (kappa values $0.61,0.67,0.69$ and 0.66 , respectively); moderate for eccentric lesions (kappa 0.45); fair for media ruptures (kappa 0.25 ); and poor for lipid deposits and plaque ruptures (kappa 0.0 and 0.04 , respectively). Differences for the arc of normal wall and hard lesion were not significant, but were for dissection. There were no significant interobserver differences between area measurements. The coefficient of variation for free lumen area and media-bounded area before PTA was $17.2 \%$ and $10.5 \%$ and after PTA $11.2 \%$ and $9.2 \%$, respectively. This study identified the intravascular ultrasound parameters that are reproducible.
\end{abstract}

Key Words: Intravascular ultrasound, Reproducibility, Peripheral arteries, Balloon angioplasty.

\section{INTRODUCTION}

From intravascular ultrasound studies performed in vitro it was shown that the characteristics of both the atherosclerotic lesion and arterial wall seen on ultrasound relate to the histologic composition (Gussenhoven et al. 1989a,b; Mallery et al. 1990; Potkin et al. 1990; Tobis et al. 1989). The location and thickness of the lesion measured from the ultrasound images correlated well with the data derived from the corresponding histologic sections. Intra- and interobserver agreement of in vitro and in vivo images obtained prior co intervention from normal and diseased peripheral arteries have shown good correlation for quantitative measurements (di Mario et al. 1992; Wenguang et al. 1990, 1991). However, a systematic analysis of the interobserver agreement on the qualitative and quantitative intravascular ultrasound parameters obtained clinically prior to and following balloon angioplasty in peripheral arteries is lacking.

Address for correspondence: Dr. E. J. Gussenhoven, Erasmus University Rotterdam (Ee 2312), P.O. Box 1738, 3000 DR Rotterdam, The Netherlands. E-mail: lugt@tch.fgg.eur.nl
The purpose of this study was to determine the interobserver agreement on qualitative and quantitative intravascular ultrasound parameters obtained in a clinical setting before and after balloon angioplasty of the superficial femoral artery.

\section{METHODS}

\section{Intravascular ultrasound imaging}

In 38 patients ( 29 men, 9 women; ages $64 \pm 10$ years), intravascular ultrasound was performed before and after balloon angioplasty for treatment of occlusive superficial femoral artery disease. Patients were included in the study after written informed consent. Intravascular ultrasound studies were performed using mechanical systems based on a single ultrasound element $(30 \mathrm{MHz})$. Nine patients were studied with the CVIS "Insight"' system (Sunnyvale, CA ) using a 4.3F flexible catheter containing a rotating mirror; 29 patients were studied with the Du-MED system (Rotterdam, The Netherlands) using a $4.3 F$ flexible catheter ("Princeps") containing a rotating element. Before and immediately after balloon angioplasty the intravas- 
cular ultrasound catheter was advanced distally and cross-sections were recorded during pull-back of the catheter together with their frame number. Images for analysis were recorded with $1-\mathrm{cm}$ intervals. Under fluoroscopic control, the location of the intravascular ultrasound catheter was systematically compared with the radiopaque ruler; its location was indicated manually on the video monitor (Gussenhoven et al. 1991). The resulting images were displayed on the monitor via a video-scanned memory and stored on an S-VHS recorder. The videotapes were reviewed and, according to the protocol, all cross-sections within the treated segment (interval $1 \mathrm{~cm}$ ) obtained before and after balloon angioplasty underwent qualitative and quantitative analysis.

Two experienced observers from different institutions took part in the data acquisition in their own hospitals. The cross-sections selected by one observer were subsequently analyzed by the other observer using the unique frame number as marker for the crosssections.

First, the intravascular ultrasound parameters used in this study were defined. To get acquainted with the application of these definitions the selected intravascular ultrasound cross-sections ( 64 before and 38 after) of the first five patients (not included in this study) were analyzed by the two observers after which disagreement was discussed and solved by consensus. Subsequently, the selected intravascular ultrasound cross-sections of each patient in this study (median five per patient; range 2-12) were independently analyzed by the two observers $(n=192$ before and $n=$ 212 after balloon angioplasty).

\section{Qualitative analysis}

Cross-sections were assessed for lesion topography and morphology seen before balloon angioplasty and for presence of vascular damage seen after intervention. Criteria for evaluating the qualitative features with intravascular ultrasound have been described previously (Gerritsen et al. 1993; Gussenhoven et al. 1991, The et al. 1992). Lesion topography may be either eccentric or concentric. Lesion morphology may include fibromuscular/fibrous/thrombus (echo-soft), calcification (echo-hard with shadowing) and/or lipid deposit (echo-poor). These morphologic features may be present simultaneously in one cross-section. After balloon angioplasty, the presence of dissection, plaque rupture and media rupture was documented. 'These features may be present simultaneously in one cross-section. In the presence of one or more of these features "vascular damage" was scored.

The arc of normal wall, hard lesion and dissection that subtends the circumference was graded by visual estimation according to the hours of a clock with the center of the vessel as reference ( $1 \mathrm{~h}$ corresponding to $30^{\circ}$ of a sector). In the presence of multiple calcium deposits and or dissections, the total sum was calculated. For instance, a lesion may involve 5 adjacent hours of the circumference leaving the remaining 7 disease-free (normal).

\section{Quantitative analysis}

Quantitative measurements were performed using a computer-based analysis system (Wenguang et al. 1990, 1991). Analysis included measurement of free lumen area and media-bounded area (Fig. 1). In the absence of a visible tunica media on intravascular ultrasound, the adventitia was used as reference.

During off-line quantitative analysis of the free lumen area, it was experienced that the presence of strong scattering due to blood may cause difficulties in discrimination of the luminal houndary. To diminish this problem, echo images were replayed several times on real-time on an additional video monitor to distinguish the luminal boundary from the echoes of bloodscatter.

\section{Definitions}

Eccentric lesion was involved when part of the vessel wall was disease-free. The vessel wall was judged to be normal when the lesion thickness was $0.5 \mathrm{~mm}$ or less. Soft lesion was defined as having a homogeneous or bright echostructure superimposed on the arterial wall without shadowing. Hard lesion was recognized by the presence of a bright echostructure superimposed on the arterial wall casting peripheral shadowing. Lipid deposit was defined as a hypoechoic region inside a lcsion.

Dissection was defined as the presence of a tear in the intimal surface separating the lesion from the underlying arterial wall. Plaque rupture was defined as a radial tear in the intimal surface perpendicular to the arterial wall. Media rupture (i.e., internal elastic lamina rupture) was defined as an interruption in the internal elastic lamina which exposes the hypoechoic media or hyperechoic adventitia to arterial lumen. Vascular damage was defined as the presence of dissection, plaque rupture and/or media rupture.

Free lumen area $\left(\mathrm{mm}^{2}\right)$ was defined as the area encompassed by the inner boundary of the intimal surface (characterized also by the presence of blood).

Media-bounded area $\left(\mathrm{mm}^{2}\right)$ was defined as the native vessel area bounded by the inner medial border.

\section{Statistical unalysis}

First, the interobserver reproducibility for the presence or absence of each qualitative parameter was expressed as unweighted Cohen's kappa coefficient (Cohen 


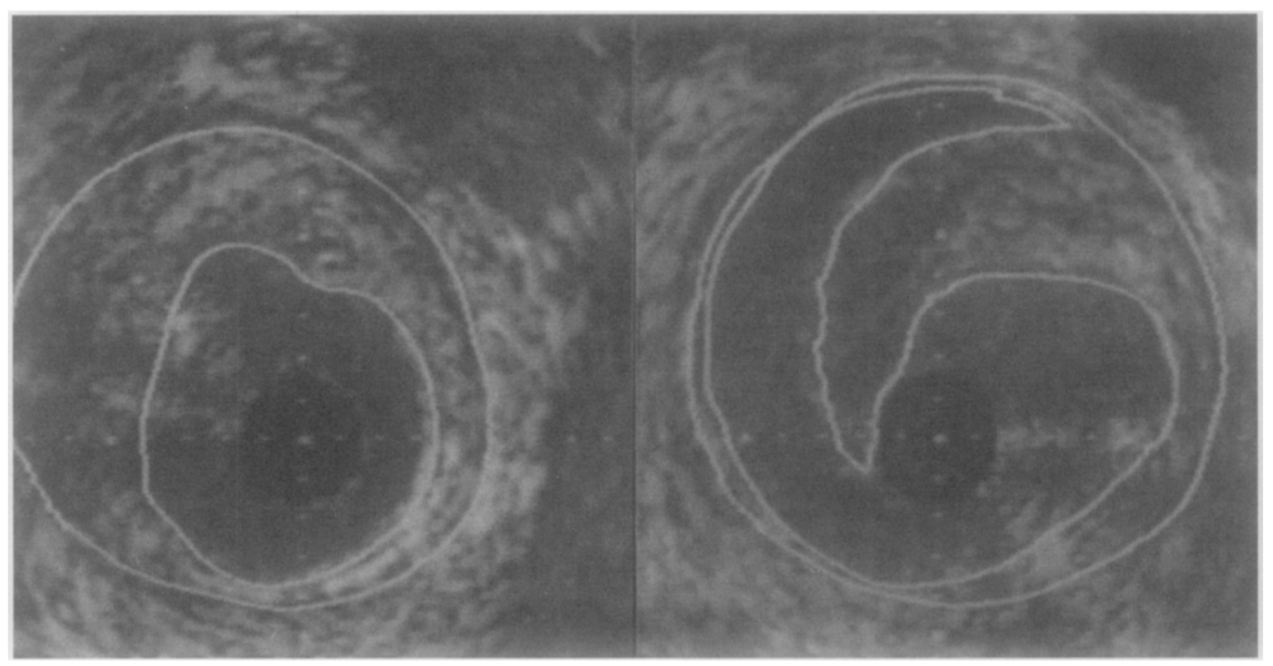

Fig. 1. Corresponding intravascular ultrasound cross-sections obtained from a patient hefore and after halloon angioplasty (PTA) of the superficial femoral artery using the CVIS system. Before PTA (left), a soft eccentric lesion was involved; after PTA (right) a dissection was diagnosed. The extent of the arterial wall that was diseasefree was $1 \mathrm{~h} \mathrm{(} 6 \mathrm{o}^{\prime} \mathrm{clock}$ ) and of the dissection, $4 \mathrm{~h}$. The intravascular ultrasound cross-sections are contour-traced off-line for free lumen area (inner contour) and media-bounded area (outer contour). Calibration $=0.5 \mathrm{~mm}$.

1960). Kappa values range from -1 to +1 . Kappa equals +1 when there is complete agreement between two observers. Kappa equals 0 when observed agreement is equal to that expected by chance alone. When agreement is less than chance agrecment then kappa has a negative value. Kappa values below 0.20 represent poor agreement; values ranging from 0.20 to 0.40 represent fair agreement; values from 0.40 to 0.60 represent moderate agreement; those from 0.60 to 0.80 represent good agreement; and values greater than 0.80 represent very good agreement (Landis and Koch 1977).

Second, the total percentage of agreement was calculated as a percentage of all comparisons for each diagnostic category that showed agreement, regarding either the absence or the presence of the diagnostic category.

Third, to describe the agreement between the observers in the extent of the qualitative parameters and the measurements of the free lumen area and mediabounded area, mean and standard deviations of the paired differences between the two observers were given. For data related to the extent of qualitative parameters, images in which both observers agreed on the presence of these features were considered. The Student's $t$-test for paired observations was used to test whether there were systematic differences between observers. The degree of interobserver variation is presented with a coefficient of variation defined as the standard deviation of the paired difference divided by the mean of the absolute value. A $p$-value $<0.05$ was considered statistically significant.

Fourth, the absolute paired differences in quantita- tive measurements between the systems used were compared using the Wilcoxon two-sample test.

\section{RESULTS}

The interobserver reproducibility of the qualitative intravascular ultrasound parameters obtained before and after balloon angioplasty are summarized in Table 1. There was good agreement for soft lesion, hard lesion, dissection and for vascular damage (Figs. 1-3). Moderate agreement was observed for eccentric lesion. Fair agreement was found for media rupture. Poor agreement was found for lipid deposit and for plaque rupture.

Table 1. Kappa values and total percentage of agreement (TA) for all intravascular ultrasound qualitative parameters seen before $(n=192)$ and after $(n=212)$ balloon angioplasty (PTA).

\begin{tabular}{lll}
\hline & Kappa & TA \\
\hline Before PTA & & \\
Eccentric lesion & 0.45 & $73 \%$ \\
Soft lesion & 0.61 & $94 \%$ \\
Hard lesion & 0.67 & $85 \%$ \\
Lipid deposit & 0.00 & $95 \%$ \\
After PTA & & \\
Dissection & 0.69 & $85 \%$ \\
Plaque rupture & 0.04 & $71 \%$ \\
Media rupture & 0.25 & $86 \%$ \\
Vascular damage & 0.66 & $83 \%$ \\
\hline
\end{tabular}

$n=$ Number of cross-sections. 


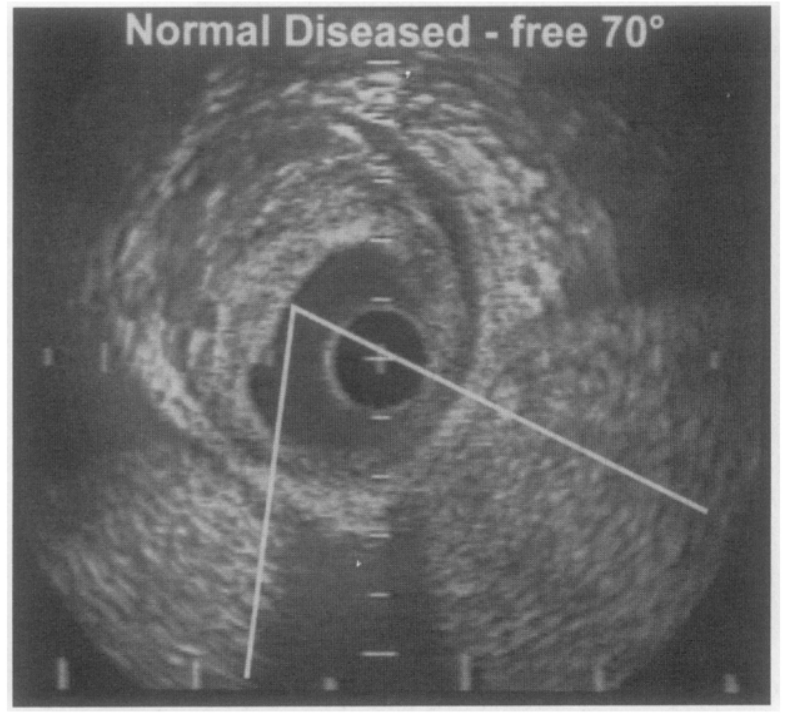

Fig. 2. Intravascular ultrasound cross-section obtained from a patient before balloon angioplasty of the superficial femoral artery using the Du-MED system. The lesion involved was eccentric and soft in nature. The hypoechoic region seen at 10-11 o'clock may suggest the presence of lipid deposit, but could also be an artifact of attenuation. The arc of disease-free arterial wall is $2 \mathrm{~h}$ ( 5 o'clock). $+=$ catheter; calibration $=1 \mathrm{~mm}$.

Differences between the two observers were not significant for the arc of nomal wall and hard lesion; differences for the arc of dissection were significant (Table 2).

In 165 of the 192 cross-sections obtained before

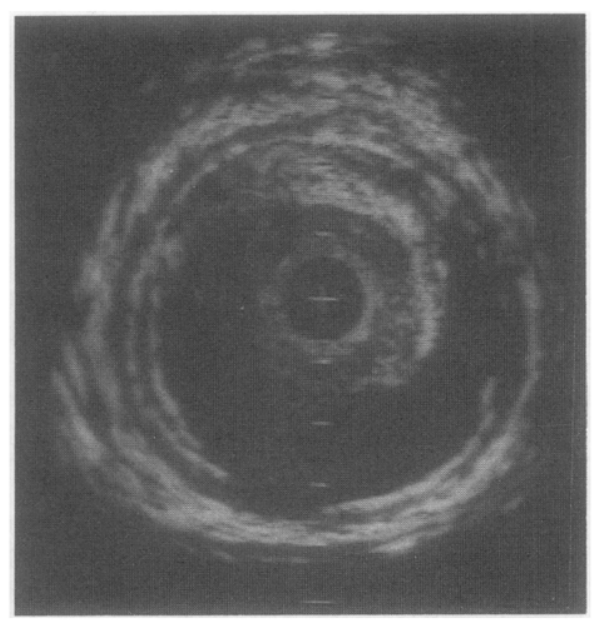

Fig. 3. Intravascular ultrasound cross-section ohtained from a patient after balloon angioplasty of the superficial femoral attery. The lesion involved, eccentric and soft in nature, became dissected from the arterial wall. Both the extent of the dissection (between 2 and 4 o'clock) and media rupture were $2 \mathrm{~h}$ (at 3 and at 7 o'clock). $+=$ catheter; calibration $=1 \mathrm{~mm}$.
Table 2. Paired difference and coefficient of variation between the two observers of qualitative parameters (normal wall, hard lesion and dissection) expressed as arc in hours.

\begin{tabular}{lcrr}
\hline & $n$ & Paired difference & $\begin{array}{r}\text { Coefficient } \\
\text { of variation }\end{array}$ \\
\hline Normal wall & 75 & $0.2 \pm 2.1$ (NS) & $5 \%$ \\
Hard lesion & 50 & $-0.2 \pm 1.1$ (NS) & $34 \%$ \\
Dissection & 70 & $0.6 \pm 1.6(\mathrm{~S})$ & $21 \%$ \\
\hline
\end{tabular}

Data represent mean and standard deviation; $n=$ number of crosssections; NS - not significant; $\mathbf{S}=$ - significant.

intervention, and 177 of the 212 cross-sections obtained after intervention, media-bounded area could be assessed. In 62 cross-sections ( 27 before and 35 after intervention), media-bounded area could not be assessed due to calcification and/or inadequate image quality. There was no significant interobserver bias for free lumen area and media-bounded area calculated before and after balloon angioplasty (Table 3 ). The coefficient of interobserver variation was $17.2 \%$ and $10.5 \%$ for free lumen area and media-bounded area, respectively, before intervention and $11.2 \%$ and $9.2 \%$ for free lumen area and media-bounded area, respectively, after intervention. Results of the Wilcoxon twosample test showed that the mean absolute difference of the free lumen area and media-bounded area obtained with the two intravascular ultrasound systems used was not significantly different.

\section{DISCUSSION}

Reliable distinction between different qualitative features and assessment of quantitative parameters with intravascular ultrasound both before and after intervention is important for clinical practice. Tenaglia et al. (1992) were the first to report on the observer agreement on qualitative analysis of intracoronary ultrasound images. The reported kappa values were 0.70

Table 3. Paired differences and coefficient of variation between the two observers of the area measurements obtained before and after balloon angioplasty (PTA).

\begin{tabular}{lcc}
\hline & Paired difference & $\begin{array}{c}\text { Coefficient } \\
\text { of variation }\end{array}$ \\
\hline Before PTA & & \\
Free lumen area & $+0.22 \pm 2.07 \mathrm{~mm}^{2}$ (NS) & $17.2 \%$ \\
Media-bounded area & $-0.02 \pm 3.05 \mathrm{~mm}^{2}$ (NS) & $10.5 \%$ \\
& & \\
After PTA & $+0.03 \pm 1.94 \mathrm{~mm}^{2}$ (NS) & $11.2 \%$ \\
$\quad$ Free lumen area & & $9.2 \%$ \\
Media-bounded area & $-0.11 \pm 3.03 \mathrm{~mm}^{2}$ (NS) & $9.2 \%$ \\
\hline
\end{tabular}

Data represent mean and standard deviation. NS = not significant. 
for hard, 0.42 for eccentric and 0.66 for dissection, respectively. Similar kappa values were found by $\mathrm{Pe}-$ ters et al. (1994) for calcium (0.9) and for eccentric lesion (0.5). In addition, they reported low kappa values for intimal thickening (0.3), and for lipid $(-0.1)$. Furthermore, Hausmann et al. (1994) established that intravascular ultrasound parameters assessed qualitatively (presence of normal wall, plaque or calcium) showed excellent agreement within and between observers; quantitative parameters also showed low variability. Unfortunately, important issues such as vascular damage and quantitative analyses seen after intervention wcre not part of their study (Hausmann ct al. 994; Peters et al. 1994).

The present study with intravascular ultrasound performed in peripheral arteries revealed a high reproducibility for the detection of soft lesion, hard lesion, dissection and vascular damage. We postulate that the moderate agreement found for eccentric lesions (kappa value 0.45 ) may be due to the definition used: "eccentric lesion was involved if the lesion measured 0.5 nm or less at the normal vessel wall." This arbitrary criterion assessed by visual estimation might have inthenced the outcome of data.

Unequivocally, the diagnostic accuracy for lipid deposit was poor (see also Fig. 2). Although the total agreement was high, the kappa value was low. Total agreement was high because both observers had not seen lipid deposits inside the lesion in most of the cross-sections. However, as they did not agree in the few cases in which they scored lipid deposit, kappa value was therefore low. The accuracy of intravascular ultrasound to detect lipid deposit in coronary arteries may be hampered because echolucent areas may alternatively represent acoustic drop-out, a dissection, or a thick media (Peters et al. 1994). In addition to lesion composition, gain setting of the system and angle of incidence are known to be important determinants that influence the echogenicity of a lesion (di Mario et al. 1992; Potkin et al. 1990). Recent in vitro studies showed that the sensitivity for lipid detection was low $(<30 \%)$ and the specificity of this finding was high $(>80 \%$ ) (Gussenhoven et al. 1994; Sechtem et al. 1993 ). For this reason, we are reluctant in diagnosing lipid deposit (i.e., "instable plaque") with the current intravascular ultrasound systems.

After balloon angioplasty, we found a good agreement for dissection and vascular damage; similar kappa values for dissection (0.66) were reported by Tenaglia et al. (1992). However, agreement for media rupture was fair and, for plaque rupture, poor. This may be related to the resolution of the intravascular ultrasound system used. Whereas a dissection may present as a large well-delineated feature, both plaque rupture and media rupture were usually small and may potentially be overlooked.

It is also noteworthy, that differences for the arc of normal wall and hard lesion were not significant, but were for dissection (see Table 2). The reason for this difference may be related to the visual estimation of the arc of the dissection. It is not uncommon that the arc of dissection represents a sum of dissections seen in one cross-section. We assume that, if a protractor was used, a more reproducible measure might have been obtained.

Finally, the reproducibility of the quantitative data in this study was high for frec lumen arca and mediabounded area calculated before and after balloon angioplasty. Moreover, data obtained with the two ultrasound systems used were not significantly different both before and after intervention. This observation is of crucial importance given the fact that recent intravascular ultrasound studies in both coronary and peripheral arteries have shown that quantitative assessment of free lumen area and diameter seen following balloon angioplasty are predictive factors of failure (Gussenhoven et al. 1995; Jain et al. 1994).

It should be noted that a limitation of this study is the low number of cross-sections available with the CVIS system. In addition, the analyses for this study were executed off-line. It is a challenge to know whether similar results can be obtained on-line, for example, in "battle-field conditions" for decisionmaking during intervention.

In view of the clinical application of intravascular ultrasound, it is important to know which intravascular ultrasound parameters are reproducible for clinical decisionmaking.

\section{SUMMARY}

This study indicates that highly reproducible intravascular ultrasound parameters include: (1) soft lesion, hard lesion and dissection; (2) the arc of normal wall and hard lesion; and (3) the quantitative assessment of free lumen area and media-bounded area both before and after balloon angioplasty.

\section{REFERENCES}

Cohen JA. A coefficient of agreement for nominal scales. Educ Psychol Meas 1960:20:37-46.

Gerritsen GP, Gussenhoven EJ, The SHK, Pieterman H, Lugt van der A. Intravascular ultrasonography before and after intervention: In vivo comparison with angingraphy. I Vasc Surg 1993;80:31 40.

Gussenhoven EJ, Essed CE, Lancée CT, Mastik F, Frietman P. Arterial wall characteristics determined by intravascular ultrasound imaging: an in vitro study. J Am Coll Cardiol $1989 a ; 14: 947-952$.

Gussenhoven WJ, Essed CE, Frietman P, Mastik F, Lancée CT Intravascular echographic assessment of vessel wall characteris- 
tics: a correlation with histology. Int J Card Imag 1989b;4:105116.

Gussenhoven EJ, Frietman PAV, The SHK, Suylen van RJ, Egmond van FC. Assessment of medial thinning in atherosclerosis by intravascular ultrasound. Am J Cardiol 1991;68:1625-1632.

Gussenhoven EJ, Lugt van der A, Strijen van M, Stigter C, Li W. Intracoronary ultrasound before and after balloon angioplasty: An in vitro validation with histology [abstract]. Eur Heart $\mathbf{J}$ 1994; 15:209.

Gussenhoven EJ, Lugt van der A, The SHK, Pasterkamp G. Berg van den FG. Intravascular ultrasound predictors of outcome after peripheral balloon angioplasty. Eur J Endovasc Surg 1995; 10:279-288.

Hausmann D, Lundkvist AS, Friedrich GJ, Mullen WL. Fitzgerald PJ. Intracoronary ultrasound imaging: Intraobserver and interobserver variability of morphometric measurements. Am Heart $\mathbf{J}$ 1994; 128:674-680.

Jain SP, Jain A, Collins TJ, Ramee SR, White CJ. Predictors of restenosis: A morphometric and quantitative evaluation by intravascular ultrasound. Am IIeart J 1994; 128:664-673.

Landis JR, Koch GG. The measurement of observer agreement for categorical data. Biometrics 1977;33:671-679.

Mallery JA, ''obis JM, Griffith J, Gessert J, McKae M. Assessment of normal and atherosclerotic arterial wall thickness with an intravascular ultrasound imaging catheter. Am Heart J 1990; 119:1392-1400.

di Mario C, The SHK, Madretsma S, Suylen van RJ, Wilson RA. Detection and characterization of vascular lesions by intravascu- lar ultrasound. An in-vitro correlative study with histology. J Am Soc Echocardiol 1992;5:135-146.

Peters RIG, Erbel R Ge I, Hillige HT, I inker DT. Observer agreement on validation of intracoronary ultrasound imaging. Thesis, University of Amsterdam, 1994:87-98.

Potkin BN, Bartorelli AL, Gessert JM, Neville RF, Almagor Y. Coronary artery imaging with intravascular high-frequency ultrasound. Circulation 1990;81:1575-1585.

Sechtem U, Arnold G, Kcweloh T, Casper C, Curtius JM. In-VitroDiagnose der koronaren Plaquemorphologie mit Intravasculärem Ultraschall: Vergleich mit Histopatologischen Befunden, Zeitschrift für Kardiologie 1993;82:618-627.

Tenaglia AN, Buller CE, Kisslo K, Phillips HR, Stack RS. Intracoronary ultrasound predictors of adverse outcomes after coronary artery interventions. J Am Coll Cardiol 1992;20:1385-1390.

The SHK, Gussenhoven EJ, Zhong Y, Li W, van Egmond FC. The effect of balloon angioplasty on the femoral artery evaluated with intravascular ultrasound imaging. Circulation 1992;86:483493.

Tobis JM, Mallery JA, Gessert J, Griffith J, Mahon D. Intravascular ultrasound cross-sectional arterial imaging before and after balloon angioplasty in vitro. Circulation 1989;80:873-882.

Wenguang L, Gussenhoven WJ, Bosch JG, Mastik F, Reiber JHC. A computer-aided analysis system for the quantitative assessment of intravascular ultrasound images. Proc Comput Cardiol 1990: $333-336$.

Wenguang L, Gussenhoven WJ. Zhong Y, The SHK, Di Mario C. Validation of quantitative analysis of intravascular ultrasound images. Int J Card Imag 1991:6:247-253. 